STUDI

FRANCESI

\section{Studi Francesi}

Rivista quadrimestrale fondata da Franco Simone

174 (LVIII | III) | 2014

Varia

Dupaty et l'Italie des voyageurs sensibles, études réunies par Jan Herman, Kris Peeters et Paul Pelckmans

\title{
Eszter Kovács
}

\section{CpenEdition}

Journals

Édition électronique

URL : http://journals.openedition.org/studifrancesi/1174

DOI : 10.4000/studifrancesi. 1174

ISSN : 2421-5856

Éditeur

Rosenberg \& Sellier

Édition imprimée

Date de publication : 1 novembre 2014

Pagination : 599-600

ISSN : 0039-2944

\section{Référence électronique}

Eszter Kovács, « Dupaty et l'Italie des voyageurs sensibles, études réunies par Jan Herman, Kris Peeters et Paul Pelckmans », Studi Francesi [En ligne], 174 (LVIII | III) | 2014, mis en ligne le 01 novembre 2014, consulté le 18 septembre 2020. URL : http://journals.openedition.org/studifrancesi/1174 ; DOI :

https://doi.org/10.4000/studifrancesi. 1174

Ce document a été généré automatiquement le 18 septembre 2020.

\section{cc) (†) $\odot$}

Studi Francesi è distribuita con Licenza Creative Commons Attribuzione - Non commerciale - Non opere derivate 4.0 Internazionale. 


\title{
Dupaty et l'Italie des voyageurs sensibles, études réunies par Jan Herman, Kris Peeters et Paul Pelckmans
}

\author{
Eszter Kovács
}

\section{RÉFÉRENCE}

Dupaty et l'Italie des voyageurs sensibles, études réunies par Jan HERMAN, Kris PEETERS et Paul PELCKMANS, Amsterdam - New York, Rodopi, 2012, pp. 278.

1 Le volume réunit les actes du colloque qui a eu lieu à Rome en 2011. Le sujet - le voyage sensible, dont Dupaty est considéré comme un des premiers représentants - est en soi très large; la problématique est délimitée par l'espace - l'Italie - et l'époque - les auteurs contemporains ou postérieurs de quelques décennies aux Lettres sur l'Italie, publiées en 1788. Les premiers articles sont consacrés à Dupaty, d'autres aux auteurs moins connus, deux articles traitent de la fiction qui se déroule en Italie (Corinne, Valérie ). Parmi les ouvrages analysés, il y a des lettres et des journaux de voyage, des romans, des textes publiés ou restés inédits, avec ou sans prétention littéraire. Leur caractéristique plus ou moins commune est le regard subjectif, la sensibilité au paysage, aux œuvres d'art, au passé, à la précarité du sort humain, à ses propres sentiments.

2 Paul Pelckmans souligne dans l'Introduction (pp. 7-10) la nouveauté de Dupaty: c'est le moi du voyageur qui s'impose. Dans Dupaty et les réformes italiennes (pp. 11-23), Laurence MACÉ observe la réflexion sur les lois criminelles - une réflexion originale, quoique, à son avis, l'image des réformes soit partielle et peut-être partiale. Dans L'errance sensible de Dupaty (pp. 25-35), Sylviane LEONI s'intéresse au «regard errant» du voyageur qui caractérise les Lettres. Dans Dupaty et Gênes: quelques réactions artistiques 
(pp. 37-43), Giulia SAVIO examine les impressions du voyageur sur le patrimoine de la ville; on sent toutefois un peu que l'article est détaché d'un travail plus large. L'article de Mladen kozuL, Dupaty et la politique (pp. 45-56), observe la réflexion sur l'inégalité devant la loi, sur les tensions socio-politiques, ainsi que l'influence des Lettres sur Sade, qui en était lecteur. Vient ensuite une série d'articles consacrés aux autres voyageurs. Comme le remarque Jean-Daniel CANDAUX dans Lalande et Dupaty en mains: le «Voyage en Italie» du Genevois Jacques Le Fort (pp. 57-67). Le Fort marque un certain désaccord avec l'opinion de Dupaty déjà l'année de la parution des Lettres. Dans l'article Un Grand Tour interrompu: le récit de voyage inédit du sculpteur Philippe-Laurent Roland (pp. 69-87), Cécilie CHAMPY présente un voyage lié à la campagne de Bonaparte, à la recherche des œuvres d'art à emporter: il s'agit de notes et d'esquisses sans prétention littéraire mais elles témoignent d'une sensibilité au paysage caractéristique de l'époque. L'article de Margherita BRECCIA FRATADOCCHI (pp. 89-95), Pour un tourisme durable ante litteram. L'«Itinerario italiano», est consacré à un guide pratique, sans valeur littéraire mais familier au nombre de voyageurs. Dans Le Tivoli des âmes sensibles (pp. 97-111), Catriona SETH observe le rôle du site chez plusieurs voyageurs $d u$ XVIII ${ }^{e}$-XIX ${ }^{e}$ siècles, parmi lesquels il y a des peintres et des écrivains. L'article Les Antiquaires. Une petite comédie et le "grand théâtre» d'Italie chez le Marquis de Sade (pp. 113-123) de Valerio CANTAFIO CASAMAGGI analyse les références italiennes dans cette pièce, influencée par Goldoni; la liberté semble être le plus grand attrait du pays pour Sade. Le voyage en Italie dans les «Lettres» de la Princesse de Gonzague (pp. 125-141) de Stéphanie BURETTE nous fait connaître une contemporaine de Dupaty, dont les Lettres témoignent d'une sensibilité «féminine» face au paysage et aux œuvres humaines. Irini APOSTOLOU, dans Dupaty et Creuzé de Lesser à la recherche des beaux-arts (pp. 143-156), analyse le Voyage en Italie et en Sicile du dernier et constate que le regard subjectif relie les deux textes malgré les différences. Selon Emmanuelle TABET - Des «Lettres sur l'Italie» de Dupaty au «Voyage en Italie» de Chateaubriand (pp. 157-169) - l'influence de l'état d'âme du voyageur sur sa perception est encore plus forte chez Chateaubriand que chez Dupaty, il va plus loin dans l'exaltation de ses sentiments. Jan HERMAN observe dans Les années de pèlerinage de Mme de Krüdener: «Valérie» et l'Italie (pp. 171-188) un texte entre le roman épistolaire et le journal intime, dans lequel l'Italie déclenche un amour malheureux et exalté. Dans L'émoi de l'artiste en voyage. Émotions et expériences sensibles d'un architecte en Italie (pp. 189-205), Sébastien CHAUFFOUR présente le récit de Jean-Jacques Huvé, rédigé trente ans après le voyage à partir de notes, réécrites pour faire ressortir l'unicité de ses expériences. Sophie GUERMÈs avance dans Poésie, nature, vérité: Bonstetten sur les traces de Virgile (pp. 207-219) qu'il s'agit d'une démarche particulière qui tente d'effacer la distinction entre fiction et vérité, textes antiques et pays moderne. Dans "Corinne», l'Italie et la mort (pp. 221-236), P. PELCKMANS examine ce qui suit le modèle du roman sentimental et, au contraire, ce que l'on peut considérer novateur chez Mme de Staël. La mort semble être le dénouement nécessaire de l'amour; Pelckmans suggère qu'un certain «culte» de la mortalité italien y contribue. Dans Les «Lettres» du Marquis de Foresta: un regard mélancolique sur la Sicile (pp. 237-246), Giuseppina TARDANICO présente un regard plus ou moins typique sur les ruines que l'idée de la fragilité et de la vanité domine. La sensibilité n'est pas toujours évidente: comme le constate Sabine VERHULST dans L'itinéraire sensible d'un Brugeois éclairé (pp. 247-259) André Jacopssen oscille entre un inventaire et un journal plus personnel. J. HERMAN conclut dans L'Italie des âmes sensibles et les trois esprits de Dupaty (pp. 261-272) que l'ouvrage du Président est une 
référence pour certains et une attitude déjà dépassée pour d'autres: le voyage sensible évolue pendant la période en question.

3 Le voyage en Italie et la transformation du récit de voyage durant cette époque sont deux sujets souvent étudiés, le volume y apporte toutefois de nouveaux éléments. Le voyage sensible s'impose comme un modèle et, comme tout modèle, il peut être rejeté. Le climat, le paysage, l'histoire antique et moderne y jouent un rôle certain ainsi que la coexistence de la magnificence et de la pauvreté, ce qui amène les voyageurs et les protagonistes fictifs en voyage à une réflexion sur leurs propres impressions et réactions. Il serait d'ailleurs très difficile de définir ou de délimiter exactement ce phénomène. Certes, cela n'était pas l'objectif du volume, dont les auteurs ont pourtant réussi à en contourner et éclairer certains points. 